\title{
HLA Matching
}

National Cancer Institute

\section{Source}

National Cancer Institute. HLA Matching. NCI Thesaurus. Code C68707.

A process in which blood or tissue samples are analyzed to determine their human leukocyte antigen (HLA) profiles so that transplant materials can be transfered into a patient with the same or similar profile. 